1. E. D. Rainville, Special Functions, Macmillan Company, New York, 1960.

2. J. L. Lavoie, "On inverses of finite segments of the generalized Hilbert matrix," $M T A C$, v. 18, 1964, p. 141-143.

3. G. Pólya \& G. Szego, Aufgaben und Lehrsätze aus der Analysis, vol. 2 (reprinted), Dover Publications, New York, 1945.

4. A. R. COLlaR, "On the reciprocal of a segment of a generalized Hilbert matrix," Proc. Cambridge Philos. Soc., v. 47, 1951, p. 11-17.

5. W. N. BAILEY, "On the sum of a terminating ${ }_{3} F_{2}(1)$," Quart. J. Math. Oxford Ser. (2) v. 4,1953 , p. $237-240$.

\title{
Conditional Least Squares Polynomial Approximation
}

\author{
By R. W. Klopfenstein
}

There are many motivations for the development of least squares polynomial approximations to sets of data. If the data is empirical, the motivation may be the smoothing out of empirical errors to obtain a representation superior in accuracy to the original data. Or if the data is in principle exact, the motivation may be to obtain a compact approximate representation for the data. In the case of data having widely variable character, it is often expedient to segment it and produce distinct polynomial representations in different ranges of the independent variable.

In many cases, it is necessary to introduce constraints on the least square approximation problem. These may occur, for example, in connection with data for which certain properties are known exactly from the underlying physical or mathematical model. They may occur also where data is to be fitted in several separate ranges and it is desired to preserve certain continuity properties from one segment of the representation to the next.

When the least square polynomials are being provided via the normal equations [1], it is often reasonably straightforward to solve the constraint equations analytically for one or more of the undetermined coefficients and insert these into the set of linear algebraic normal equations. Use of the normal equations is, however, extremely wasteful of both storage and computing time. In addition, the normal equations are notoriously poorly conditioned so that one is rarely successful in producing least square polynomials beyond the fifth or sixth degree with single precision calculations.

The least squares algorithm via orthogonal polynomials [2] is vastly superior in almost every respect. This is especially true when the Lanczos three-term recursion [3] is incorporated in the process. In this case, if the resulting polynomial is to be used solely for function evaluation purposes, it is not even necessary to produce the explicit resulting polynomial with the inherent resulting rounding problems.

It is the purpose of this note to describe a simple transformation that will permit the solution of the least squares approximation problem subject to a class of constraint conditions. This transformation results from the generalization of an approach suggested by Hamming [4]. The transformed problem is of standard least squares type without constraints and may be solved through standard algorithms for this purpose.

Received April 22, 1964. 
We consider a set of datum points and associated weights $\left\{x_{k}, y_{k}, w_{k}\right\}$, and require that the sum

$$
S_{N}=\sum_{k=1}^{m} w_{k}\left\{y_{k}-Q_{N}\left(x_{k}\right)\right\}^{2}
$$

be minimized where $Q_{N}(x)$ is a polynomial of $N$ th degree and there are $l$ prescribed constraints on $Q_{N}(x)$ at one or more points $x_{j}$ of the form

$$
Q_{N}{ }^{(r)}\left(x_{j}\right)=b_{r j}, \quad r=0,1, \cdots, R_{j}
$$

The $x_{j}$ may be points on which the data is specified, $x_{k}$, but are not necessarily these. It is to be noted that all of the $b_{r j}$ must be specified. We define $P_{l-1}(x)$ to be the polynomial of $(l-1)$ st degree satisfying these constraint conditions and set

$$
Q_{N}(x)=P_{l-1}(x)+\Pi_{l}(x) Q_{N-l}(x),
$$

where

$$
\Pi_{l}(x)=\prod_{j=1}^{J}\left(x-x_{j}\right)^{1+R_{j}}
$$

We now minimize the sum

$$
S_{N}=\sum_{k=1}^{m} w_{k}^{\prime}\left\{y_{k}^{\prime}-Q_{N-l}\left(x_{k}\right)\right\}^{2}
$$

where

$$
y_{k}^{\prime}=\frac{y_{k}-P_{l-1}\left(x_{k}\right)}{\Pi_{l}\left(x_{k}\right)}, \quad \text { and } w_{k}{ }^{\prime}=w_{k} \Pi_{l}{ }^{2}\left(x_{k}\right) \text {. }
$$

The $Q_{N}(x)$ so obtained satisfies the constraint conditions (2) by definition. Direct substitution shows that the minimization problem (4) is equivalent to that of (1). If any of the $x_{j}$ are identical with one of the data points, $x_{k}$, these points are eliminated from both (1) and (4) since the constraint conditions (2) impose a fixed contribution to $S_{N}$ corresponding to these points. Therefore, the problem of zero divisors in (4) does not arise.

It may be appropriate to exhibit a few specific examples of the above. Suppose first that we have a set of data from experiment and it is known from the physical problem that the function represented must be zero when the independent variable, $x$, is zero. In this case,

$$
\begin{aligned}
P_{0}(x) & =0, \\
\Pi_{1}(x) & =x, \\
y_{k}^{\prime} & =\frac{y_{k}}{x_{k}}, \quad \text { and } \\
w_{k}{ }^{\prime} & =w_{k} x_{k}^{2} .
\end{aligned}
$$

If the fitting polynomial is constrained to take on prescribed values at the two end-points, 


$$
\begin{aligned}
Q_{N}\left(x_{1}\right) & =b_{1}, \\
Q_{N}\left(x_{m}\right) & =b_{m},
\end{aligned}
$$

we have

$$
\begin{aligned}
& P_{1}(x)=\frac{b_{1}\left(x-x_{m}\right)-b_{m}\left(x-x_{1}\right)}{x_{1}-x_{m}}, \\
& \Pi_{2}(x)=\left(x-x_{1}\right)\left(x-x_{m}\right),
\end{aligned}
$$

and the end-points are eliminated from the sum in (4).

If the fitting polynomial is constrained to have a specified value, $a$, and slope, $b$, when the independent variable is zero, then

$$
\begin{aligned}
P_{1}(x) & =a+b x, \\
\Pi_{2}(x) & =x^{2}, \\
y_{k}{ }^{\prime} & =\frac{y_{k}}{x_{k}{ }^{2}}, \text { and } \\
w_{k}{ }^{\prime} & =w_{k} x_{k}{ }^{4} .
\end{aligned}
$$

The utility of the above in segmented curve fitting is apparent. Having obtained an approximating polynomial in the first range of the independent variable, we may require the approximating polynomial for the second range to agree with that of the first at the point where transition between representations is to occur. We may, indeed, require continuity of one or more derivatives at this point and/or agreement on more points if desired.

An effective technique for producing polynomial approximations for mathematical functions is to normalize the range of the independent variable to $[-1,1]$ and then do a least squares approximation over the values of the independent variable corresponding to the zeros of a Tschebycheff polynomial of suitable degree [5]. This produces a nearly optimal polynomial approximation over the range. In some cases, criteria other than minimum absolute error may be relevant. Consider, for example, the approximation of

$$
y=\sin \left(\frac{\pi}{4} x\right), \quad-1 \leqq x \leqq 1 .
$$

In addition to minimizing the absolute error we may want to retain significance (small relative errors) in the vicinity of zero argument. This may be accomplished by adding the constraints

$$
\begin{aligned}
y(0) & =0, \quad \text { and } \\
y^{\prime}(0) & =\frac{\pi}{4} .
\end{aligned}
$$

This is readily accomplished through the transformations indicated in (7).

In summary, a simple transformation has been described which permits the adding of constraints to the least squares approximation problem. The transformed 
problem is again of standard least squares form. Flexibility in the application of least squares techniques is therefore substantially enhanced.

RCA Laboratories

Princeton, New Jersey

1. F. B. Hildebrand, Introduction to Numerical Analysis, McGraw-Hill Book Co., New York, 1956, p. 261-269.

2. F. B. Hildebrand, op. cit., p. 287-288.

3. A. S. Housenolder, Principles of Numerical Analysis, McGraw-Hill Book Co., New York, 1953, p. 221 .

4. R. W. Hamming, Numerical Methods for Scientists and Engineers, McGraw-Hill Book Co., New York, 1962, p. 242.

5. NBS Applied Mathematics Series, No. 9, Tables of Chebyshev Polynomials $S_{n}(\alpha)$ and $C_{n}(\alpha)$, U. S. Govt. Printing Office, Washington, D. C., 1952; Introduction.

\section{Improved Asymptotic Expansion for the Error Function with Imaginary Argument}

\section{By D. van Z. Wadsworth}

The well-known asymptotic approximation to the error function can be markedly improved, for the case with imaginary argument, by adding a simple correction term as shown below. The improved analytic approximation was needed in connection with the analysis of spacecraft and ICBM re-entry trajectories.

By definition* the error function with imaginary argument $i x$ where $x$ is real is

$$
\operatorname{erf}(i x)=i \int_{0}^{x} e^{s^{2}} d s=\frac{i}{2} \int_{0}^{x^{2}} t^{-1 / 2} e^{t} d t=\frac{i}{2} \int_{L} t^{-1 / 2} e^{t} d t-\frac{\pi^{1 / 2}}{2} .
$$

The branch cut for $t^{-1 / 2}$ extends along the negative imaginary axis of the $t$ plane and the Riemann sheet is chosen for which $t^{-1 / 2}$ is positive on the positive real axis. The path of integration $L$ goes from $-\infty$ to $x^{2}$ as shown in Figure 1. Repeated partial integration of the infinite integral yields $-i$ erf $(i x)=E_{n}(x)+e_{n}(x)$ where

$$
E_{n}(x)=\frac{x^{-1} e^{x^{2}}}{2} \sum_{0}^{n} r_{m} x^{-2 m}
$$

is the asymptotic approximation for the interval $\left(n-\frac{1}{2}\right) \leqq x^{2}<\left(n+\frac{1}{2}\right)$ and

$$
e_{n}(x)=\frac{1}{2} r_{n+1} \int_{L} t^{-n-3 / 2} e^{t} d t+\frac{i}{2} \pi^{1 / 2}
$$

is the error of the asymptotic approximation. The coefficient $r_{n}=2^{-2 n}(2 n) ! / n !$.

The integral in equation (3) is equivalent to a line integral on the segment $\left[-\infty,-x^{2}\right]$ and an integral on the semi-circle joining $-x^{2}$ and $x^{2}$. If we let $x^{2} \exp (i \pi-i \varphi)=t$ in the latter integral we obtain

$$
\begin{aligned}
\int_{L} t^{-n-3 / 2} e^{t} d t=(-)^{n+1} x^{-2 n-1} \int_{0}^{\pi} \exp \left[-x^{2} \cos \varphi+\right. & \left.i x^{2} \sin \varphi+i\left(n+\frac{1}{2}\right) \varphi\right] d \varphi \\
& +(-)^{n+1} i \int_{x^{2}}^{\infty} t^{-n-3 / 2} e^{-t} d t
\end{aligned}
$$

Received April 20, 1964.

* This definition differs by a factor of $2 \pi^{-1 / 2}$ from that given by some authors. 